\title{
A note on the existence and multiplicity of solutions for sublinear fractional problems
}

\section{Yongqiang $\mathrm{Fu}^{*}$ (1)}

\author{
"Correspondence: \\ fuyongqiang@hit.edu.cn \\ Department of Mathematics, Harbin \\ Institute of Technology, Harbin \\ 150001, P.R. China
}

\begin{abstract}
In this paper, we study the existence of weak solutions for fractional $p$-Laplacian equations with sublinear growth and oscillatory behavior as the following

$$
\begin{array}{ll}
\mathcal{L}_{K}^{p} u=\lambda f(x, u) & \text { in } \Omega, \\
u=0 & \text { in } \mathbb{R}^{N} \backslash \Omega,
\end{array}
$$

where $\mathcal{L}_{K}^{p}$ is a nonlocal operator with singular kernel, $\Omega$ is an open bounded smooth domain of $\mathbb{R}^{N}$. Our purpose is to generalize the known results for fractional Laplacian equations to fractional $p$-Laplacian equations.
\end{abstract}

MSC: 35R11; 35A15; 35J60; 47G20

Keywords: fractional $p$-Laplacian problem; variational method; multiple solutions

\section{Introduction}

Recently, a great attention has been devoted to the research of problems involving fractional and nonlocal operators. This type of operators finds many applications in a lot of fields, such as continuum mechanics, phase transition phenomena, population dynamics and game theory, as they are the typical outcome of stochastic stabilization of Lévy processes; see, for instance, [1-4] and the references therein. There are many works on nonlocal fractional operators and their applications which are very interesting; we refer the interested reader to [5-14] and the references therein. Here we want to generalize the multiplicity existence results for fractional Laplacian equations in [15] and at the same time fix some bugs there.

In this paper we deal with the following fractional problem in a bounded smooth domain $\Omega \subset \mathbb{R}^{N}$ :

$$
\begin{array}{ll}
\mathcal{L}_{K}^{p} u=\lambda f(x, u) & \text { in } \Omega, \\
u=0 & \text { in } \mathbb{R}^{N} \backslash \Omega,
\end{array}
$$

where $\lambda>0,1<p<+\infty . \mathcal{L}_{K}^{p}$ is a nonlocal fractional operator defined as follows:

$$
\mathcal{L}_{K}^{p} \varphi=2 \lim _{\varepsilon \rightarrow 0} \int_{\mathbb{R}^{N} \backslash B_{\varepsilon}(x)}|\varphi(x)-\varphi(y)|^{p-2}(\varphi(x)-\varphi(y)) K(x-y) d y,
$$

(c) The Author(s) 2017. This article is distributed under the terms of the Creative Commons Attribution 4.0 International License (http://creativecommons.org/licenses/by/4.0/), which permits unrestricted use, distribution, and reproduction in any medium, provided you give appropriate credit to the original author(s) and the source, provide a link to the Creative Commons license, and indicate if changes were made. 
provided that the limit exists and $K$ is a measurable function having the following property:

$$
\begin{cases}\gamma K \in L^{1}\left(\mathbb{R}^{N}\right) & \text { where } \gamma(x)=\min \left\{|x|^{p}, 1\right\} \\ \text { there exist } \lambda, \Lambda>0, & \\ \text { such that } \lambda<K(x)|x|^{N+p s}<\Lambda & \text { for any } x \in \mathbb{R}^{N} \backslash\{0\}, \\ K(x)=K(-x) & \text { for any } x \in \mathbb{R}^{N} \backslash\{0\}\end{cases}
$$

Throughout this paper we assume $N>p s$ with $s \in(0,1)$. A typical example for $K$ is the singular kernel $|x|^{-(N+p s)}$, in which case problem (1.1) becomes

$$
\begin{array}{ll}
(-\Delta)_{p}^{s} u=\lambda f(x, u) & \text { in } \Omega, \\
u=0 & \text { in } \mathbb{R}^{N} \backslash \Omega,
\end{array}
$$

where $(-\Delta)_{p}^{s}$ is the fractional $p$-Laplacian operator defined as

$$
(-\Delta)_{p}^{s} \varphi(x)=2 \lim _{\varepsilon \rightarrow 0} \int_{\mathbb{R}^{N} \backslash B_{\varepsilon}(x)} \frac{|\varphi(x)-\varphi(y)|^{p-2}(\varphi(x)-\varphi(y))}{|x-y|^{N+s p}} d y
$$

for all $x \in \mathbb{R}^{N}$. In the case $p=2$, problem (1.3) becomes the fractional Laplacian problem

$$
\begin{array}{ll}
(-\Delta)^{s} u=\lambda f(x, u) & \text { in } \Omega, \\
u=0 & \text { in } \mathbb{R}^{N} \backslash \Omega .
\end{array}
$$

Following $[16,17]$, we present the main structural assumptions on the nonlinear term $f$.

$\left(f_{1}\right) f \in C\left(\mathbb{R}^{\mathbb{N}} \times \mathbb{R}\right), f(x, 0)=0, \liminf _{t \rightarrow 0} \frac{f(x, t)}{|t|^{p-2} t}>-\infty$.

$\left(f_{2}\right) \lim _{|t| \rightarrow \infty} \frac{f(x, t)}{|t|^{p-1}}=0$ uniformly in $x$.

$\left(f_{3}\right)$ There exist $t^{-}<0$ and $t^{+}>0$ such that $\min \left\{f(x, t):(x, t) \in \bar{\Omega} \times\left\{t^{-}\right\}\right\}>0$ and $\max \{f(x, t)$ : $\left.(x, t) \in \bar{\Omega} \times\left\{t^{+}\right\}\right\}<0$.

$\left(f_{4}\right)$ There exist $\hat{t}^{-}$and $\hat{t}^{+}$, with $\hat{t}^{-}<t^{-}$and $\hat{t}^{+}>t^{+}$, such that

$$
\min \left\{F(x, t):(x, t) \in \bar{\Omega} \times\left\{\hat{t}^{-}, \hat{t}^{+}\right\}\right\}>\max \left\{f(x, t):(x, t) \in \bar{\Omega} \times\left[t^{-}, t^{+}\right]\right\},
$$

where $F(x, t)=\int_{0}^{t} f(x, \xi) d \xi$.

A typical example of $f$ satisfying $\left(f_{1}\right)-\left(f_{4}\right)$ is

$$
f(x, t)= \begin{cases}|t|^{p-1}(|t|-1) \operatorname{sgn}(t), & |t|<1, \\ (|t|-1)^{p-1-\varepsilon} \operatorname{sgn}(t), & |t|>1,\end{cases}
$$

where $\varepsilon$ is small.

The natural solution space of problem (1.1) is

$$
X_{0}(\Omega)=\left\{u \in X\left(\mathbb{R}^{N}\right): u=0 \text { a.e. in } \mathbb{R}^{N} \backslash \Omega\right\},
$$


where $X\left(\mathbb{R}^{N}\right)$ is the fractional Sobolev space given by

$$
X\left(\mathbb{R}^{N}\right)=\left\{u \in L^{p}\left(\mathbb{R}^{N}\right):[u]_{s, p}<\infty\right\}, \quad[u]_{s, p}=\left(\iint_{\mathbb{R}^{2 N}}|u(x)-u(y)|^{p} K(x-y) d x d y\right)^{1 / p}
$$

and endowed with the norm

$$
\|u\|_{X\left(\mathbb{R}^{N}\right)}=\left(\|u\|_{L^{p}\left(\mathbb{R}^{N)}\right.}^{p}+[u]_{s, p}^{p}\right)^{1 / p}
$$

For the basic properties of fractional Sobolev spaces, we refer to [18]. We seek the solutions of (1.1) as the critical points of the functional given by

$$
I_{\lambda}(u)=\frac{1}{p}[u]_{s, p}^{p}-\lambda \int_{\Omega} F(x, u) d x
$$

Set

$$
\alpha_{\lambda}=\left.\inf I_{\lambda}\right|_{\left[t^{-}, t^{+}\right]}
$$

where $t^{-}$and $t^{+}$are the numbers given in $\left(f_{3}\right)$.

Theorem 1.1 Assume that $\left(f_{1}\right)-\left(f_{4}\right)$ hold. Then there exists $\Lambda>0$ such that, for every $\lambda \geq$ $\Lambda$, problem (1.1) admits two positive, two negative and two sign-changing solutions in $Y \backslash$ $\left[t^{-}, t^{+}\right]$, where $Y:=X_{0}(\Omega) \cap C^{\alpha / 2}(\bar{\Omega})$.

Here, for two real numbers $t<s$, the symbol $[t, s]$ denotes either an order interval in $Y$ or a usual interval in $\mathbb{R}$. In this paper there will be no ambiguous meaning concerning this symbol, for example, in Theorem $1.1 Y \backslash\left[t^{-}, t^{+}\right]$denotes the difference of $Y$ and $\left[t^{-}, t^{+}\right]$, where $\left[t^{-}, t^{+}\right]$is an order interval in $Y$.

Theorem 1.2 Assume that $\left(f_{1}\right)-\left(f_{4}\right)$ hold and $f(x, t)$ is odd with respect to $t$. Then, for every $k \in \mathbb{N}$, there exists positive $\Lambda_{k}$ such that, for every $\lambda \geq \Lambda_{k}$, problem (1.1) admits a signchanging solution $u_{i}$ with $I_{\lambda}\left(u_{i}\right)<\alpha_{\lambda}$ and a sign-changing solution $v_{i}$ with $I_{\lambda}\left(v_{i}\right) \geq \alpha_{\lambda}$, where $i=1, \ldots, n$.

Next consider the case that $f(x, t)$ is not odd with respect to $t$, but oscillates around 0 in the following manner.

$\left(f_{5}\right)$ There exist $t_{i}^{-}$and $t_{i}^{+}, i=1, \ldots, n$, with $t_{n}^{-}<\cdots<t_{1}^{-}<0<t_{1}^{+}<\cdots<t_{n}^{+}$, such that $\min \left\{f(x, t):(x, t) \in \bar{\Omega} \times\left\{t_{i}^{-}\right\}\right\}>0>\max \left\{f(x, t):(x, t) \in \bar{\Omega} \times\left\{t_{i}^{+}\right\}\right\}$for all $i=1, \ldots, n$.

$\left(f_{6}\right) \min \left\{F(x, t):(x, t) \in \bar{\Omega} \times\left\{t_{i}^{-}, t_{i}^{+}\right\}\right\}>\max \left\{F(x, t):(x, t) \in \bar{\Omega} \times\left[t_{i-1}^{-}, t_{i-1}^{+}\right]\right\}$for $i=2, \ldots, n$.

Theorem 1.3 Assume that $\left(f_{1}\right)-\left(f_{2}\right)$ and $\left(f_{5}\right)-\left(f_{6}\right)$ hold. Then there exists $\Lambda>0$ such that, for every $\lambda \geq \Lambda$, problem (1.1) admits positive solutions $u_{i 1}, u_{i 2}$, negative solutions $v_{i 1}, v_{i 2}$, and sign-changing solutions $w_{i 1}, w_{i 2}$ in $\left[t_{i+1}^{-}, t_{i+1}^{+}\right] \backslash\left[t_{i}^{-}, t_{i}^{+}\right], i=1, \ldots, n-1$.

At last, we consider the case of $f$ having infinitely many oscillations in both $(-\infty, 0)$ and $(0,+\infty)$. 
$\left(f_{7}\right)$ There exist a decreasing (an increasing) sequence $\left(t_{i}^{-}\right)_{i}$ and an increasing (a decreasing) sequence $\left(t_{i}^{+}\right)_{i}$ such that $t_{i}^{-}<0<t_{i}^{+}$, and $f\left(x, t_{i}^{+}\right)<0<f\left(x, t_{i}^{-}\right)$and $\max \{F(x, t):(x, t) \in$ $\left.\bar{\Omega} \times\left[t_{i-1}^{-}, t_{i-1}^{+}\right]\right\}<\min \left\{F(x, t):(x, t) \in \bar{\Omega} \times\left\{t_{i}^{-}, t_{i}^{+}\right\}\right\}$for every $i \in \mathbb{N}$.

Theorem 1.4 Assume that $\left(f_{1}\right)-\left(f_{2}\right)$ and $\left(f_{7}\right)$ hold. Then, for every arbitrarily chosen $k \in \mathbb{N}$, there exists $\Lambda_{k}>0$ such that, for every $\lambda \geq \Lambda_{k}$, problem (1.1) admits at least $2 k$ positive, $2 k$ negative, and $2 k$ sign-changing solutions.

Theorem 1.4 is immediate in view of Theorem 1.3.

Remark 1.1 Bartsch and Liu obtained Theorems 1.1-1.4 for $p$-Laplacian Dirichlet problems in [16]. Fu and Pucci obtained Theorems 1.1-1.4 for fractional Laplacian Dirichlet problems in [15]. Theorems 1.1-1.4 above are generalizations of the corresponding results in [15].

This article is organized as follows. In Section 2, we introduce the fractional Sobolev spaces and some preliminary results. In Section 3, in a suitably chosen framework, we verify that the conditions in the abstract critical point theorems in [16] are satisfied, then we generalize the existence of multiplicity solutions for fractional Laplacian problems to the one for fractional $p$-Laplacian problems.

\section{Preliminaries}

Let us now recall some inequalities.

Lemma 2.1 (See [19]) There exist positive constants $C_{1}$ - $C_{4}$ such that, for all $\xi, \eta \in \mathbb{R}^{N}$,

$$
\begin{aligned}
& \left.|| \xi\right|^{p-2} \xi-|\eta|^{p-2} \eta\left|\leq C_{1}(|\xi|+|\eta|)^{p-2}\right| \xi-\eta \mid, \\
& \left(|\xi|^{p-2} \xi-|\eta|^{p-2} \eta\right)(\xi-\eta) \geq C_{2}(|\xi|+|\eta|)^{p-2}|\xi-\eta|^{2}, \\
& \left.|| \xi\right|^{p-2} \xi-|\eta|^{p-2} \eta\left|\leq C_{3}\right| \xi-\left.\eta\right|^{p-1}, \quad \text { if } 1<p \leq 2, \\
& \left(|\xi|^{p-2} \xi-|\eta|^{p-2} \eta\right)(\xi-\eta) \geq C_{4}|\xi-\eta|^{p}, \quad \text { if } p>2 .
\end{aligned}
$$

The Hölder regularity up to the boundary, strong maximum principles and the Hopf lemma are important in the proof of our results.

Lemma 2.2 (See [20]) Let $u \in X_{0}$ satisfy $\left|\mathcal{L}_{K}^{p} u\right| \leq K$ weakly in $\Omega$ for some $K>0$. Then

$$
|u(x)| \leq\left(C_{\Omega} K\right)^{1 / p-1} \delta(x)^{s} \quad \text { a.e. in } \Omega
$$

for some $C_{\Omega}=C(N, p, s, \Omega), \delta(x)=\operatorname{dist}\left(x, \Omega^{C}\right)$. Furthermore, there exists $\alpha \in(0, s]$ such that, for all weak solutions $u \in X_{0}$ of problem $(1.1), u \in C^{\alpha}(\bar{\Omega})$ and

$$
\|u\|_{C^{\alpha}(\bar{\Omega})} \leq C_{\Omega}\|f\|_{L_{\infty}(\Omega)}^{1 / p-1} .
$$

Lemma 2.3 (See [8]) If $u \in X_{0}$ is such that $u(x) \geq 0$ a.e. in $\Omega$ and

$$
\iint_{\mathbb{R}^{2 N}}|u(x)-u(y)|^{p-2}(u(x)-u(y))(\varphi(x)-\varphi(y)) K(x-y) d x d y \geq 0
$$

for each $\varphi \in X_{0}, \varphi(x) \geq 0$ a.e. in $\Omega$, then $u(x)>0$ a.e. in $\Omega$. 
Let $s \in(0,1), p \in(1, \infty)$. Consider the problem

$$
\left\{\begin{array}{l}
(-\Delta)_{p}^{s} u=c(x)|u|^{p-2} u \quad \text { in } \Omega \\
u=0 \quad \text { in } \mathbb{R}^{N} \backslash \Omega
\end{array}\right.
$$

Lemma 2.4 (see [21]) Let $\Omega$ be bounded and satisfy the interior ball condition on $\partial \Omega$, $0 \geq c \in C(\bar{\Omega})$, and $u \in X_{0}$ be a weak solution of (2.5), then either $u=0$ a.e. in $\mathbb{R}^{N}$ or

$$
\liminf _{B_{R} \ni x \rightarrow x_{0}} \frac{u(x)}{\delta_{R}(x)^{s}}>0
$$

where $\delta_{R}(x)=\operatorname{dist}\left(x, B_{R}^{C}\right)$.

In the sequel, for any fixed parameter $\lambda>0$, the space $X_{0}(\Omega)$ is endowed with the norm

$$
\|u\|_{\lambda}=\left([u]_{s, p}^{p}+\lambda m\|u\|_{L^{p}(\Omega)}^{p}\right)^{1 / p}
$$

and, for brevity, we put $X_{\lambda}=\left(X_{0}(\Omega),\|\cdot\|_{\lambda}\right)$. The number $m>0$ will be determined in Section 3. $Y=X_{\lambda} \cap C^{\alpha / 2}(\bar{\Omega})$ is endowed with the $C^{\alpha / 2}$-norm and $Z=X_{\lambda} \cap C^{\alpha}(\bar{\Omega})$ with the $C^{\alpha}$-norm, where $\alpha>0$ is from Lemma 2.2 .

\section{Multiplicity of weak solutions}

We say that $u \in X_{\lambda}$ is a (weak) solution of problem (1.1) if

$$
\langle u, \varphi\rangle_{s, p}=\lambda \int_{\Omega} f(x, u) \varphi(x) d x
$$

holds for any $\varphi \in X_{\lambda}$, where

$$
\langle u, \varphi\rangle_{s, p}=\iint_{\mathbb{R}^{2 N}}|u(x)-u(y)|^{p-2}(u(x)-u(y))(\varphi(x)-\varphi(y)) K(x-y) d x d y .
$$

Fix $\lambda>0$ and $m>0$. Define $\mathcal{L}(v)=\mathcal{L}_{K}^{p} v+\lambda m|v|^{p-2} v$ and

$$
A_{\lambda}: X_{\lambda} \rightarrow X_{\lambda}, \quad A_{\lambda}(u)=v,
$$

where $v$ is the solution of the linear problem

$$
\begin{cases}\mathcal{L}_{K}^{p} \nu+\lambda m|v|^{p-2} v=\lambda\left(f(x, u)+m|u|^{p-2} u\right) & \text { in } \Omega, \\ v=0 & \text { in } \mathbb{R}^{N} \backslash \Omega,\end{cases}
$$

which is uniquely determined.

In the following we give a series of lemmas to show that the conditions in the abstract critical point theorems in [16] are satisfied. First we study the properties of the operator $A_{\lambda}$.

Lemma 3.1 $A_{\lambda} \in C\left(X_{\lambda}, X_{\lambda}\right)$ is well defined and $A_{\lambda}(Y) \subset Z$. 
Proof First, from

$$
\langle\mathcal{L}(v), v\rangle_{X_{\lambda}^{*}, X_{\lambda}}=\left\langle\mathcal{L}_{K}^{p} v+\lambda m|v|^{p-2} v, v\right\rangle_{X_{\lambda}^{*}, X_{\lambda}}=[v]_{s, p}^{p}+\lambda m \int_{\Omega}|v|^{p} d x
$$

we know that

$$
\lim _{\|v\|_{X_{\lambda}} \rightarrow \infty} \frac{\langle\mathcal{L}(v), v\rangle_{X_{\lambda}^{*}, X_{\lambda}}}{\|v\|_{X_{\lambda}}}=\infty
$$

i.e., the operator $\mathcal{L}$ is coercive. Second, from

$$
\begin{aligned}
& \left\langle\mathcal{L}\left(v_{1}\right)-\mathcal{L}\left(v_{2}\right), v_{1}-v_{2}\right\rangle_{X_{\lambda}^{*}, X_{\lambda}} \\
& =\iint_{\mathbb{R}^{2 N}}\left(\left|v_{1}(x)-v_{1}(y)\right|^{p-2}\left(v_{1}(x)-v_{1}(y)\right)-\left|v_{2}(x)-v_{2}(y)\right|^{p-2}\right. \\
& \left.\quad \times\left(v_{2}(x)-v_{2}(y)\right)\right)\left(\left(v_{1}(x)-v_{1}(y)\right)-\left(v_{2}(x)-v_{2}(y)\right)\right) K(x-y) d x d y \\
& \quad+\lambda m \int_{\Omega}\left(\left|v_{1}\right|^{p-2} v_{1}-\left|v_{2}\right|^{p-2} v_{2}\right)\left(v_{1}-v_{2}\right) d x
\end{aligned}
$$

$>0$,

whenever $v_{1} \neq v_{2}$, we get that $\mathcal{L}$ is monotone. It is easy to show that $\mathcal{L}$ is weakly continuous. Thus, by the monotone operator theory, see [22, 23], we conclude that problem (3.1) has a unique (weak) solution in $X_{\lambda}$. Therefore, $A_{\lambda}$ is well defined.

Next we prove that $A_{\lambda} \in C\left(X_{\lambda}, X_{\lambda}\right)$. By $\left(f_{1}\right)$ and $\left(f_{2}\right)$, we have $|f(x, u)| \leq C\left(|u|^{p-1}+1\right)$ for some constant $C$ depending only on $f$. Suppose that $u_{n} \rightarrow u$ in $X_{\lambda}$. Then $\left(u_{n}\right)_{n}$ is bounded in $X_{\lambda}$. By (3.1), Hölder's and Young's inequalities, we have

$$
\begin{aligned}
{\left[v_{n}\right]_{s, p}^{p}+\lambda m \int_{\Omega}\left|v_{n}\right|^{p} d x } & =\int_{\Omega} f\left(x, u_{n}\right) v_{n} d x+\lambda m \int_{\Omega}\left|u_{n}\right|^{p-2} u_{n} v_{n} d x \\
& \leq C\left(\left\|u_{n}\right\|_{L^{p}(\Omega)}^{p-1}+1\right)\left\|v_{n}\right\|_{L^{p}(\Omega)} \\
& \leq C \varepsilon\left\|v_{n}\right\|_{L^{p}(\Omega)}^{p}+C(\varepsilon)\left(\left\|u_{n}\right\|_{L^{p}(\Omega)}^{p}+1\right) .
\end{aligned}
$$

Taking $\varepsilon=\lambda m / 2 C$, we conclude that $\left(v_{n}\right)_{n}$ is bounded in $X_{\lambda}$. Hence, $\left(v_{n}\right)_{n}$ admits a weakly convergent subsequence, still denoted by $\left(v_{n}\right)_{n}$. Suppose that $v_{n} \rightarrow v$ weakly in $X_{\lambda}$. By Lemma 2.5 in [7], we have $v_{n} \rightarrow v$ in $L^{p}(\Omega)$ since $\Omega$ is bounded. By [24], we get $f\left(x, u_{n}\right) \rightarrow$ $f(x, u)$ in $L^{p}(\Omega)$ by going to a further subsequence if necessary. By (3.1) and Lemma 2.1, using an argument similar to (3.2), we also have

$$
\begin{aligned}
\int_{\Omega} & \left(\lambda f(x, u)+\lambda m|u|^{p-2} u-\lambda f\left(x, u_{n}\right)-\lambda m\left|u_{n}\right|^{p-2} u_{n}\right)\left(A_{\lambda}(u)-v_{n}\right) d x \\
= & \left\langle\mathcal{L}_{K}^{p} A_{\lambda}(u)+\lambda m\left|A_{\lambda}(u)\right|^{p-2} A_{\lambda}(u)-\mathcal{L}_{K}^{p} v_{n}-\lambda m\left|v_{n}\right|^{p-2} v_{n}, A_{\lambda}(u)-v_{n}\right\rangle_{X_{\lambda}^{*}, X_{\lambda}} \\
= & \iint_{\mathbb{R}^{2 N}}\left(|v(x)-v(y)|^{p-2}(v(x)-v(y))-\left|v_{n}(x)-v_{n}(y)\right|^{p-2}\right. \\
& \left.\times\left(v_{n}(x)-v_{n}(y)\right)\right)\left((v(x)-v(y))-\left(v_{n}(x)-v_{n}(y)\right)\right) K(x-y) d x d y
\end{aligned}
$$




$$
\begin{aligned}
& +\lambda m \int_{\Omega}\left(|v|^{p-2} v-\left|v_{n}\right|^{p-2} v_{n}\right)\left(v-v_{n}\right) d x \\
\geq & C \iint_{\mathbb{R}^{2 N}}\left(|v(x)-v(y)|+\left|v_{n}(x)-v_{n}(y)\right|\right)^{p-2} \\
& \times\left|(v(x)-v(y))-\left(v_{n}(x)-v_{n}(y)\right)\right|^{2} K(x-y) d x d y \\
& +C \lambda m \int_{\Omega}\left(|v|+\left|v_{n}\right|\right)^{p-2}\left|v-v_{n}\right|^{2} d x \\
\geq & C\left(\left[v-v_{n}\right]_{s, p}^{p}+\lambda m \int_{\Omega}\left|v-v_{n}\right|^{p} d x\right) .
\end{aligned}
$$

Letting $n \rightarrow \infty$, we obtain

$$
v_{n} \rightarrow v=A_{\lambda}(u) \quad \text { in } X_{\lambda} .
$$

This shows that $A_{\lambda} \in C\left(X_{\lambda}, X_{\lambda}\right)$, as claimed.

If $u \in Y$, then by $\left(f_{1}\right)$ and $\left(f_{2}\right)$ the solution $v$ of (3.1) is in $L^{\infty}(\Omega)$ thanks to Lemma 2.4. Therefore, $\Omega \ni x \mapsto g(x)=\lambda\left(f(x, u(x))+m|u(x)|^{p-2} u(x)\right)$ is in $L^{\infty}(\Omega)$ by $\left(f_{1}\right),\left(f_{2}\right)$ and the fact that $u \in L^{\infty}(\Omega)$. Hence, $v \in C^{\alpha}(\bar{\Omega})$ by Lemma 2.2 , since $v \in X_{\lambda}$, and so $v \in Z$ by the definition of $Z$.

Second, we show that conditions $\left(J_{1}\right)$ and $\left(J_{2}\right)$ in [16] are satisfied.

\section{Lemma 3.2}

(i) If $1<p \leq 2$, then the functional $I_{\lambda}$ satisfies

$$
\begin{aligned}
& \left\langle I_{\lambda}^{\prime}(u), u-v\right\rangle_{X_{\lambda}^{*}, X_{\lambda}} \geq C_{1}\|u-v\|_{X_{\lambda}}^{2}\left(\|u\|_{X_{\lambda}}+\|v\|_{X_{\lambda}}\right)^{p-2}, \\
& \left\|I_{\lambda}^{\prime}(u)\right\|_{X_{\lambda}^{*}} \leq C_{2}\|u-v\|_{X_{\lambda}}^{p-1} .
\end{aligned}
$$

(ii) If $p \geq 2$, then the functional $I_{\lambda}$ satisfies

$$
\begin{aligned}
& \left\langle I_{\lambda}^{\prime}(u), u-v\right\rangle_{X_{\lambda}^{*}, X_{\lambda}} \geq C_{3}\|u-v\|_{X_{\lambda}}^{p}, \\
& \left\|I_{\lambda}^{\prime}(u)\right\|_{X_{\lambda}^{*}} \leq C_{4}\|u-v\|_{X_{\lambda}}\left(\|u\|_{X_{\lambda}}+\|v\|_{X_{\lambda}}\right)^{p-2} .
\end{aligned}
$$

Proof Let $u \in X_{\lambda}$. Thus $v=A_{\lambda}(u)$ implies that

$$
\begin{aligned}
& \left\langle I_{\lambda}^{\prime}(u), u-v\right\rangle_{X_{\lambda}^{*}, X_{\lambda}} \\
& =\iint_{\mathbb{R}^{2 N}}|u(x)-u(y)|^{p-2}(u(x)-u(y))((u(x)-u(y))-(v(x)-v(y))) K(x-y) d x d y \\
& \quad-\lambda \int_{\Omega} f(x, u)(u-v) d x \\
& =\iint_{\mathbb{R}^{2 N}}\left(|u(x)-u(y)|^{p-2}(u(x)-u(y))-|v(x)-v(y)|^{p-2}(v(x)-v(y))\right) \\
& \quad \times((u(x)-u(y))-(v(x)-v(y))) K(x-y) d x d y \\
& \quad+\lambda m \int_{\Omega}\left(|u|^{p-2} u-|v|^{p-2} v\right)(u-v) d x .
\end{aligned}
$$


If $p \geq 2$, it follows by Lemma 2.1 that

$$
\left\langle I_{\lambda}^{\prime}(u), u-v\right\rangle_{X_{\lambda}^{*}, X_{\lambda}} \geq C_{3}\|u-v\|_{X_{\lambda}}^{p} .
$$

If $1<p \leq 2$, then from Lemma 2.1 we have

$$
\begin{aligned}
& \left\langle I_{\lambda}^{\prime}(u), u-v\right\rangle_{X_{\lambda}^{*}, X_{\lambda}} \\
& \quad \geq C_{2} \iint_{\mathbb{R}^{2 N}}(|u(x)-u(y)|+|v(x)-v(y)|)^{p-2}|(u(x)-u(y))-(v(x)-v(y))|^{2} \\
& \quad \times K(x-y) d x d y+C_{2} \lambda m \int_{\Omega}(|u(x)|+|v(x)|)^{p-2}|u(x)-v(x)|^{2} d x
\end{aligned}
$$

By Hölder's inequality

$$
\|u-v\|_{X_{\lambda}}^{p} \leq C\left(C_{2}, p\right)\left\langle I_{\lambda}^{\prime}(u), u-\left.v\right|_{X_{\lambda}^{*}, X_{\lambda}} ^{\frac{p}{2}}\left(\|u\|_{X_{\lambda}}+\|v\|_{X_{\lambda}}\right)^{p\left(1-\frac{p}{2}\right)}\right.
$$

and therefore

$$
\left\langle I_{\lambda}^{\prime}(u), u-v\right\rangle_{X_{\lambda}^{*}, X_{\lambda}} \geq C_{1}\|u-v\|_{X_{\lambda}}^{2}\left(\|u\|_{X_{\lambda}}+\|v\|_{X_{\lambda}}\right)^{p-2}
$$

For $w \in X_{\lambda}$, we have

$$
\begin{aligned}
\left\langle I_{\lambda}^{\prime}(u), w\right\rangle_{X_{\lambda}^{*}, X_{\lambda}}= & \iint_{\mathbb{R}^{2 N}}|u(x)-u(y)|^{p-2}(u(x)-u(y))(w(x)-w(y)) K(x-y) d x d y \\
& -\lambda \int_{\Omega} f(x, u) w d x \\
= & \iint_{\mathbb{R}^{2 N}}\left(|u(x)-u(y)|^{p-2}(u(x)-u(y))-|v(x)-v(y)|^{p-2}(v(x)-v(y))\right) \\
& \times(w(x)-w(y)) K(x-y) d x d y+\lambda m \int_{\Omega}\left(|u|^{p-2} u-|v|^{p-2} v\right) w d x .
\end{aligned}
$$

If $1<p \leq 2$, then by Lemma 2.1

$$
\left\|I_{\lambda}^{\prime}(u)\right\|_{X_{\lambda}^{*}} \leq C_{2}\|u-v\|_{X_{\lambda}}^{p-1}
$$

If $p \geq 2$, then by Lemma 2.1 and Hölder's inequality, we have

$$
\left\|I_{\lambda}^{\prime}(u)\right\|_{X_{\lambda}^{*}} \leq C_{4}\|u-v\|_{X_{\lambda}}\left(\|u\|_{X_{\lambda}}+\|v\|_{X_{\lambda}}\right)^{p-2}
$$

Now we conclude the result.

Third, we establish the regularity of the critical points of $I_{\lambda}$.

Lemma 3.3 Let $K=\left\{u \in X_{0}(\Omega): I_{\lambda}^{\prime}(u)=0\right\}$. Then $K \subset Y$.

Proof Let $u$ be fixed in $K$. Then $u \in L^{\infty}(\Omega)$ by $\left(f_{1}\right),\left(f_{2}\right)$ and Lemma 2.2. Hence, $\Omega \ni$ $x \mapsto \lambda f(x, u(x))$ is in $L^{\infty}(\Omega)$ again by $\left(f_{1}\right),\left(f_{2}\right)$ and the fact that $u$ is in $L^{\infty}(\Omega)$. Therefore, Lemma 2.2 gives $u \in C^{\alpha}(\bar{\Omega})$ since $u \in X_{\lambda}$, and so $u \in Y$. 
Forth, we get a comparison principle for the operator $\mathcal{L}$.

Lemma 3.4 If $u, v \in X_{\lambda}$, with $v \geq u$ in $\mathbb{R}^{N} \backslash \Omega$, are such that $\mathcal{L} v \geq \mathcal{L} u$ in $\Omega$, i.e., $\langle\mathcal{L} v-$ $\mathcal{L} u, w\rangle_{X_{\lambda}^{*}, X_{\lambda}} \geq 0$ for all $w \in X_{\lambda}$, with $w \geq 0$ in $\Omega$, then $v \geq u$ in $\Omega$.

Proof Let $u, v \in X_{\lambda}$, with $v \geq u$ in $\mathbb{R}^{N} \backslash \Omega$, be such that $\mathcal{L} v \geq \mathcal{L} u$ in $\Omega$. Take $\varphi=u-v=$ $[u-v]^{+}-[u-v]^{-}, w=[u-v]^{+}=[\varphi]^{+}$. Clearly, $w \in X_{\lambda}$ and $w \geq 0$ in $\Omega$. As

$$
|b|^{p-2} b-|a|^{p-2} a=(p-1)(b-a) \int_{0}^{1}|a+t(b-a)|^{p-2} d t
$$

we have

$$
\begin{aligned}
\langle\mathcal{L} v- & \mathcal{L} u, w\rangle_{X_{\lambda}^{*}, X_{\lambda}} \\
= & \iint_{\mathbb{R}^{2 N}}(p-1)((u(y)-v(y))-(u(x)-v(x))) Q(x, y)(w(x)-w(y)) K(x-y) d x d y \\
& +\lambda m \int_{\Omega}(p-1)(v-u) R(x) w d x \\
= & \iint_{\mathbb{R}^{2 N}}(p-1) Q(x, y)\left[-\left(\varphi^{+}(x)-\varphi^{+}(y)\right)^{2}-\varphi^{-}(x) \varphi^{+}(y)-\varphi^{-}(y) \varphi^{+}(x)\right] K(x-y) d x d y \\
& -\lambda m \int_{\Omega}(p-1) R(x)\left(\varphi^{+}\right)^{2} d x \\
\geq & 0,
\end{aligned}
$$

where

$$
\begin{aligned}
& Q(x, y)=\int_{0}^{1}|(u(x)-u(y))+t((v(x)-v(y))-(u(x)-u(y)))|^{p-2} d t, \\
& R(x)=\int_{0}^{1}|u+t(v-u)|^{p-2} d t .
\end{aligned}
$$

We see that $Q(x, y) \geq 0$ and $Q(x, y)=0$ only if $v(y)=v(x)$ and $u(y)=u(x)$, and $R(x) \geq 0$. Thus $\varphi^{+}=0$ in $\Omega$, i.e., $v \geq u$ in $\Omega$.

For $u, v \in Y$, define $u \ll v$ provided $u(x)<v(x)$ for any $x \in \Omega$ and

$$
\liminf _{x=x_{0}-d v \in \Omega, d \rightarrow 0} \frac{u(x)-v(x)}{d^{\alpha / 2}}<0
$$

for any $x_{0} \in \partial \Omega$, where $v$ is the unit outward normal vector of $\partial \Omega$ at $x_{0}$. If $u \leq v$ in $\Omega$, then $[u, v]$ denotes the order interval $\{w \in Y: u \leq w \leq v\}$.

By $\left(f_{1}\right)-\left(f_{3}\right)$, we can choose suitable positive $m$ so that

$$
\begin{aligned}
& t f(x, t)+m|t|^{p}>0 \quad \text { for } t \neq 0, \\
& f(x, t)+m|t|^{p-1} t \begin{cases}>m\left|t^{-}\right|^{p-1} t^{-} & \text {for } t \geq t^{-} \\
<m\left|t^{+}\right|^{p-1} t^{+} & \text {for } t \leq t^{+}\end{cases}
\end{aligned}
$$

Fix such an $m$ from now on. 
Fifth, we give the following three technical lemmas which are necessary to verify that the conditions in the abstract critical point theorems in [16] (especially $\left(P_{1}\right)-\left(P_{3}\right)$ there) are satisfied.

Lemma 3.5 Assume that $\left(f_{3}\right)$ and $\left(f_{4}\right)$ also hold. Then there exist $\phi, \psi \in Y$ having the following properties.

(1) $t^{-} \leq \phi \ll 0 \ll \psi \leq t^{+}$.

(2) For every $u \in X_{\lambda} \cap L^{\infty}(\Omega)$ with $\phi \leq u, \phi \ll A_{\lambda}(u)$.

(3) For every $u \in X_{\lambda} \cap L^{\infty}(\Omega)$ with $u \leq \psi, A_{\lambda}(u) \ll \psi$.

(4) For every $u \in X_{\lambda} \cap L^{\infty}(\Omega)$ with $t^{-} \leq u, \phi \ll A_{\lambda}(u)$.

(5) For every $u \in X_{\lambda} \cap L^{\infty}(\Omega)$ with $u \leq t^{+}, A_{\lambda}(u) \ll \psi$.

(6) $\max \left\{F(x, t):(x, t) \in \bar{\Omega} \times\left[-\|\phi\|_{L^{\infty}(\Omega)},\|\psi\|_{L^{\infty}(\Omega)}\right]\right\}<\min \left\{F(x, t):(x, t) \in \bar{\Omega} \times\left\{s^{-}, s^{+}\right\}\right.$.

Proof Let $\psi$ be the solution of

$$
\begin{aligned}
& \mathcal{L}_{K}^{p} u+\lambda m|u|^{p-2} u=\lambda m\left|t^{+}\right|^{p-2} t^{+} \quad \text { in } \Omega, \\
& u=0 \quad \text { in } \mathbb{R}^{N} \backslash \Omega .
\end{aligned}
$$

Thus $0 \ll \psi \leq t^{+}$again by Lemma 3.4 and the strong maximum principle Lemma 2.3 since $\mathcal{L}_{K}^{p}(\psi) \geq 0$. By Lemmas 3.4 and 2.4, we also have $\liminf _{B_{R} \ni x \rightarrow x_{0}} \frac{\psi(x)}{\delta_{R}(x)^{s}}>0$. Let $\phi$ be the solution of

$$
\begin{aligned}
& \mathcal{L}_{K}^{p} u+\lambda m|u|^{p-2} u=\lambda m\left|t^{-}\right|^{p-2} t^{-} \quad \text { in } \Omega, \\
& u=0 \quad \text { in } \mathbb{R}^{N} \backslash \Omega .
\end{aligned}
$$

Therefore $t^{-} \leq \phi \ll 0$ by Lemma 3.4 and by the strong maximum principle Lemma 2.3 since $\mathcal{L}_{K}^{p}(-\phi) \geq 0$. By Lemmas 3.4 and 2.4, we also have $\liminf _{B_{R} \ni x \rightarrow x_{0}} \frac{-\varphi(x)}{\delta_{R}(x)^{s}}>0$.

Next we prove the results on $A_{\lambda}$. By $(3.3)$, we can choose $\delta \in(0,1)$ small enough such that

$$
f(x, t)+m|t|^{p-1} t \begin{cases}>\delta m\left|t^{-}\right|^{p-1} t^{-} & \text {for } t \geq t^{-} \\ <\delta m\left|t^{+}\right|^{p-1} t^{+} & \text {for } t \leq t^{+}\end{cases}
$$

Let $u \in X_{\lambda} \cap L^{\infty}(\Omega)$ such that $t^{-} \leq u$. Denote $v=A_{\lambda}(u)$. Then

$$
\mathcal{L}_{K}^{p} v+\lambda m|v|^{p-2} v=\lambda f(x, u)+\lambda m|u|^{p-2} u \geq \delta \lambda m\left|t^{-}\right|^{p-2} t^{-}=\delta\left[\mathcal{L}_{K}^{p} \phi+\lambda m|\phi|^{p-2} \phi\right] .
$$

Hence, $v \geq \delta^{\frac{1}{p-1}} \phi \gg \phi$ by Lemma 3.4.

Similarly, if $u \in X_{\lambda} \cap L^{\infty}(\Omega)$ with $u \leq t^{+}$, then $v \leq \delta^{\frac{1}{p-1}} \psi \ll \psi$. This completes the proof of (1)-(5).

Conclusion (6) follows directly from $\left(f_{2}\right)$ and properties (1)-(3).

Lemma 3.6 Suppose that $\left(f_{3}\right)$ and $\left(f_{4}\right)$ also hold. Then there exists $\Lambda>0$ such that, for every $\lambda \geq \Lambda$, there exists $h \in C([0,1], \tilde{Y})$ satisfying $h(1) \leq 0 \leq h(0)$ and

$$
\max _{0 \leq t \leq 1} J_{\lambda}(h(t))<\inf _{u \in[\phi, \psi]} J_{\lambda}(u),
$$


where $\tilde{Y}=X \cap C^{1}(\bar{\Omega})$, $\phi$ and $\psi$ are the ones in Lemma 3.5. Furthermore, if $f(x, t)$ is odd with respect to $t$, then, for every $k \in \mathbb{N}$, there exists $\Lambda_{k}>0$ such that, for every $\lambda \geq \Lambda_{k}$, there exists an odd map $h_{k} \in C\left(S^{k}, \tilde{Y}\right)$ satisfying

$$
\max _{\theta \in S^{k}} J_{\lambda}\left(h_{k}(\theta)\right)<\inf _{u \in[\phi, \psi]} J_{\lambda}(u) .
$$

The proof is a minor modification of the corresponding argument given in order to prove Lemma 3.2 of [16].

Lemma 3.7 For any bounded set $B \subset Y$ and any order interval $[\phi, \psi]$, there exist $\phi_{1}, \psi_{1} \in Y$ such that

$$
B \cup[\phi, \psi] \subset \operatorname{int}_{Y}\left[\phi_{1}, \psi_{1}\right],
$$

and

$$
A_{\lambda}(u) \in\left[\phi_{1}, \psi_{1}\right] \quad \text { for every } u \in X \text { satisfying } \phi_{1} \leq u \leq \psi_{1} .
$$

Proof By $\left(f_{2}\right)$ there exist $\varepsilon, C>0$, which depend on $\lambda$, such that

$$
\lambda|f(x, t)| \leq\left(\lambda_{1}-2 \varepsilon\right)|t|^{p-1}+C \quad \text { for every } x \in \Omega, t \in \mathbb{R},
$$

where $\lambda_{1}$ is the first eigenvalue in $\Omega$ of $\mathcal{L}_{K}^{p}$ with zero Dirichlet boundary condition. By the monotone operator theory, see [22, 23], there exists a unique solution $\psi_{1}$ to

$$
\begin{aligned}
& \mathcal{L}_{K}^{p} u-\left(\lambda_{1}-\varepsilon\right)|u|^{p-2} u=\frac{C}{\mu} \quad \text { in } \Omega, \\
& u=0 \quad \text { in } \mathbb{R}^{N} \backslash \Omega,
\end{aligned}
$$

where

$$
\mu=\frac{\lambda_{1}-2 \varepsilon+\lambda m}{\lambda_{1}-\varepsilon+\lambda m}<1
$$

Let $\phi_{1}=-\psi_{1}$. Then we get $\phi_{1} \ll 0 \ll \psi_{1}$ by Lemma 3.4 and the strong maximum principle lemma. Provided that $C>0$ is large enough, (3.6) follows.

Next, fix $u \in X_{\lambda}$ which satisfies $\phi_{1} \leq u \leq \psi_{1}$. Denote $v=A_{\lambda}(u)$. We have

$$
\begin{aligned}
\mathcal{L}_{K}^{p} \nu+\left.\lambda m|v|\right|^{p-2} v & =\lambda\left[f(x, u)+m|u|^{p-2} u\right] \\
& \leq\left(\lambda_{1}-2 \varepsilon\right)\left|\psi_{1}\right|^{p-2} \psi_{1}+C+\lambda m\left|\psi_{1}\right|^{p-2} \psi_{1} \\
& =\mu\left[\left(\lambda_{1}-\varepsilon\right)\left|\psi_{1}\right|^{p-2} \psi_{1}+\lambda m\left|\psi_{1}\right|^{p-2} \psi_{1}+C / \mu\right] \\
& =\mu\left[\mathcal{L}_{K}^{p} \psi_{1}+\lambda m\left|\psi_{1}\right|^{p-2} \psi_{1}\right] .
\end{aligned}
$$

Hence, $v \leq \mu^{\frac{1}{p-1}} \psi_{1} \ll \psi_{1}$ by Lemma 3.4. Similarly, $v \gg \phi_{1}$. This completes the proof of (3.7). 
At last, we show that $I_{\lambda}$ satisfies the Palais-Smale condition which is crucial to guaranteeing the existence of critical points.

Lemma 3.8 Let $\left(u_{n}\right)_{n} \subset X_{\lambda}$ be a Palais-Smale sequence of $I_{\lambda}$, i.e., $\left(I_{\lambda}\left(u_{n}\right)\right)_{n}$ is bounded and $I_{\lambda}^{\prime}\left(u_{n}\right) \rightarrow 0$ as $n \rightarrow \infty$. Then $\left(u_{n}\right)_{n}$ admits a strongly convergent subsequence in $X_{\lambda}$.

Proof By $\left(f_{1}\right)-\left(f_{2}\right)$, for $\varepsilon>0$ small enough,

$$
|f(x, t)| \leq C(\varepsilon)+\frac{1}{p} \varepsilon|t|^{p-1}, \quad|F(x, t)| \leq C(\varepsilon)+\varepsilon|t|^{p} .
$$

By Lemma 2.3 in [7],

$$
\|u\|_{L^{p}(\Omega)} \leq C_{1}[u]_{s, p}
$$

since $\Omega$ is bounded.

Let $\left(u_{n}\right)_{n}$ be a Palais-Smale sequence of $I_{\lambda}$ in $X_{\lambda}$. Then there exists $C>0$ such that, for all $n$,

$$
C \geq I_{\lambda}\left(u_{n}\right) \geq \frac{1}{p}\left[u_{n}\right]_{s, p}^{p}-\lambda \varepsilon\left\|u_{n}\right\|_{L^{p}(\Omega)}^{2}-C(\varepsilon)|\Omega| \geq \frac{1}{2 p}\left[u_{n}\right]_{s, p}^{p}-C_{2},
$$

where $\varepsilon=1 / 2 p C_{1}^{2} \lambda$ in (3.8). Thus $\left(u_{n}\right)_{n}$ is bounded in $X_{\lambda}$ by (3.9). So, up to a subsequence, still denoted by $\left(u_{n}\right)_{n}$, we have $u_{n} \rightarrow u$ weakly in $X_{\lambda}$. Then $\left\langle I^{\prime}\left(u_{n}\right), u_{n}-u\right\rangle \rightarrow 0$, and further we obtain

$$
\begin{aligned}
& \left\langle I^{\prime}\left(u_{n}\right), u_{n}-u\right\rangle \\
& =\iint_{\mathbb{R}^{2 N}}\left|u_{n}(x)-u_{n}(y)\right|^{p-2}\left(u_{n}(x)-u_{n}(y)\right)\left[\left(u_{n}(x)-u(x)\right)-\left(u_{n}(y)-u(y)\right)\right] \\
& \quad \times K(x-y) d x d y-\lambda \int_{\Omega} f\left(x, u_{n}\right)\left(u_{n}-u\right) d x \\
& \quad \rightarrow 0
\end{aligned}
$$

as $n \rightarrow \infty$. Moreover, by Lemma 2.5 in [7], up to a subsequence, $u_{n} \rightarrow u$ strongly in $L^{p}(\Omega)$ and a.e. in $\Omega$. Thus, $f\left(x, u_{n}\right)\left(u_{n}-u\right) \rightarrow 0$ a.e. in $\Omega$ as $n \rightarrow \infty$. It is easy to show that the sequence $\left(f\left(x, u_{n}\right)\left(u_{n}-u\right)\right)_{n}$ is uniformly bounded and equi-integrable in $L^{1}(\Omega)$. Hence, by the Vitali convergence theorem (see Rudin [25]), we get

$$
\lim _{n \rightarrow \infty} \int_{\Omega} f\left(x, u_{n}\right)\left(u_{n}-u\right) d x=0 .
$$

Therefore, by (3.10), we have

$$
\begin{aligned}
& \iint_{\mathbb{R}^{2 N}}\left|u_{n}(x)-u_{n}(y)\right|^{p-2}\left(u_{n}(x)-u_{n}(y)\right)\left[\left(u_{n}(x)-u(x)\right)-\left(u_{n}(y)-u(y)\right)\right] K(x-y) d x d y \\
& \quad \rightarrow 0
\end{aligned}
$$


as $n \rightarrow \infty$. Thus, by the weak convergence of $\left(u_{n}\right)_{n}$ in $X_{\lambda}$, we get

$$
\begin{aligned}
& \iint_{\mathbb{R}^{2 N}}\left[\left|u_{n}(x)-u_{n}(y)\right|^{p-2}\left(u_{n}(x)-u_{n}(y)\right)-|u(x)-u(y)|^{p-2}(u(x)-u(y))\right] \\
& \quad \times\left[\left(u_{n}(x)-u(x)\right)-\left(u_{n}(y)-u(y)\right)\right] K(x-y) d x d y \\
& \quad \rightarrow 0
\end{aligned}
$$

as $n \rightarrow \infty$. By Lemma 2.1, we obtain, for $p>2$,

$$
\begin{aligned}
& \iint_{\mathbb{R}^{2 N}}\left|\left(u_{n}(x)-u(x)\right)-\left(u_{n}(y)-u(y)\right)\right|^{p} K(x-y) d x d y \\
& \quad \leq C_{1} \iint_{\mathbb{R}^{2 N}}\left[\left|u_{n}(x)-u_{n}(y)\right|^{p-2}\left(u_{n}(x)-u_{n}(y)\right)-|u(x)-u(y)|^{p-2}(u(x)-u(y))\right] \\
& \quad \times\left[\left(u_{n}(x)-u(x)\right)-\left(u_{n}(y)-u(y)\right)\right] K(x-y) d x d y \\
& \quad \rightarrow 0
\end{aligned}
$$

as $n \rightarrow \infty$. For $1<p<2$, we have

$$
\begin{aligned}
& \iint_{\mathbb{R}^{2 N}}\left|\left(u_{n}(x)-u(x)\right)-\left(u_{n}(y)-u(y)\right)\right|^{p} K(x-y) d x d y \\
& \leq C_{2}\left[\iint_{\mathbb{R}^{2 N}}\left[\left|u_{n}(x)-u_{n}(y)\right|^{p-2}\left(u_{n}(x)-u_{n}(y)\right)-|u(x)-u(y)|^{p-2}(u(x)-u(y))\right]\right. \\
& \left.\quad \times\left[\left(u_{n}(x)-u(x)\right)-\left(u_{n}(y)-u(y)\right)\right] K(x-y) d x d y\right]^{p / 2} \\
& \quad \times\left[\iint_{\mathbb{R}^{2 N}} \mid\left(u_{n}(x)-u_{n}(y)|-| u(x)-\left.u(y)\right|^{p} K(x-y) d x d y\right]^{2-p / 2}\right. \\
& \leq C_{3}\left[\iint_{\mathbb{R}^{2 N}}\left[\left|u_{n}(x)-u_{n}(y)\right|^{p-2}\left(u_{n}(x)-u_{n}(y)\right)-|u(x)-u(y)|^{p-2}(u(x)-u(y))\right]\right. \\
& \left.\quad \times\left[\left(u_{n}(x)-u(x)\right)-\left(u_{n}(y)-u(y)\right)\right] K(x-y) d x d y\right]^{p / 2} \\
& \rightarrow 0
\end{aligned}
$$

as $n \rightarrow \infty$. Combining (3.11) and (3.12), we get that $u_{n} \rightarrow u$ strongly in $X_{\lambda}$ as $n \rightarrow \infty$. Therefore, $I_{\lambda}$ satisfies the (PS) condition.

Taking inspiration from [16], we apply Lemmas 3.5-3.8 in order to prove Theorems 1.11.3. Let $\operatorname{int}_{D_{1}}\left(D_{2}\right)$ refer to the $Y$-topology on $D_{1}$.

Proof of Theorem 1.1 Let $\phi, \psi$ and $\Lambda$ be the ones in Lemma 3.5 and Lemma 3.6. Fix $\lambda \geq \Lambda$. Let $h$ be the one in Lemma 3.6. Choose $B=h([0,1])$ so that $B \subset \tilde{Y} \subset Y$ by Lemma 3.6. Let the corresponding $\phi_{1}$ and $\psi_{1}$ be the ones stated in Lemma 3.7.

Define $D_{1}^{+}=\left[0, \psi_{1}\right]$ and $D_{2}^{+}=[0, \psi]$. Then

$$
\text { int }_{D_{1}^{+}} D_{2}^{+}=\{u \in Y: 0 \leq u \ll \psi\},
$$


where $\operatorname{int}_{D_{1}^{+}}\left(D_{2}^{+}\right)$refers to the $Y$-topology on $D_{1}^{+}$. By Lemmas 3.5 and 3.7 and Lemma 2.4, conditions $\left(P_{1}\right)-\left(P_{3}\right)$ in Section 2 are satisfied for $D_{2}^{+} \subset D_{1}^{+}$and $A=A_{\lambda}$. By Lemmas 3.6-3.8 and Theorem 2.1 in [17], there are two positive and two negative solutions of problem (1.1).

Choose $D_{1}=\left[\phi_{1}, \psi_{1}\right], D_{2}=\left[\phi, \psi_{1}\right], D_{3}=\left[\phi_{1}, \psi\right]$, and $D_{4}=[\phi, \psi]$. By Lemmas 3.5-3.8, $D_{i}$, $i=1, \ldots, 4$, and $A=A_{\lambda}$ satisfy all the assumptions of Theorem 2.2 in [17]. So there are two sign-changing solutions of problem (1.1).

At last, in view of (3) of Lemma 3.5, we complete the proof.

Proof of Theorem 1.2 Let $D_{i}, i=1, \ldots, 4$, be the ones in the proof of Theorem 1.1. First, in view of Lemmas 3.5-3.8, all the assumptions of Theorem 2.3 in [17] are satisfied. Second, by (2) and (3) of Lemma 3.5,

$$
\inf _{u \in\left[t^{-}, t^{+}\right]} I_{\lambda}(u) \leq \inf _{u \in D_{4}} I_{\lambda}(u)
$$

and

$$
A_{\lambda}\left(\left[t^{-}, t^{+}\right]\right) \subset \operatorname{int}_{Y}\left(D_{4}\right)
$$

Hence, (3.13)-(3.14) and Proposition 2.4 of [16] yield the result.

Proof of Theorem 1.3 Define $f_{i}(x, t), i=1, \ldots, n-1$, as in [16]. Consider

$$
\begin{cases}\mathcal{L}_{k}^{p} u=\lambda f_{i}(x, u) & \text { in } \Omega, \\ u(x)=0, & \text { in } \mathbb{R}^{N} \backslash \Omega .\end{cases}
$$

By Theorem 1.1, problem (3.15) admits two positive, two negative and two sign-changing solutions. Replacing $t^{-}, t^{+}$by $t_{i}^{-}, t_{i}^{+}$, respectively, the six solutions are outside of the order interval $\left[t_{i}^{-}, t_{i}^{+}\right]$. According to the choice of $m$, we have

$$
\left|m t_{i+1}^{-}\right|^{p-2} m t_{i+1}^{-}<f(x, t)+m t=f_{i}(x, t)+m|t|^{p-2} t<m\left|t_{i+1}^{+}\right|^{p-2} t_{i+1}^{+} \quad \text { for } t_{i+1}^{-} \leq t \leq t_{i+1}^{+} .
$$

Thus, by the definition of $f_{i}$, we get

$$
\begin{array}{ll}
\mathcal{L}_{K}^{p} u+\lambda m|u|^{p-2}>\lambda m\left|t_{i+1}^{-}\right|^{p-2} t_{i+1}^{-}=\mathcal{L}_{K}^{p} t_{i+1}^{-}+\lambda m\left|t_{i+1}^{-}\right|^{p-2} t_{i+1}^{-} & \text {in } \Omega, \\
u=0>t_{i+1}^{-} & \text {in } \mathbb{R}^{N} \backslash \Omega .
\end{array}
$$

Furthermore, by Lemma 3.4 the six solutions of problem (3.15) are inside the order interval $\left[t_{i+1}^{-}, t_{i+1}^{+}\right]$and are obviously solutions of problem (1.1). In this way, we manage to get $2(n-1)$ positive, $2(n-1)$ negative and $2(n-1)$ sign-changing solutions of problem (1.1).

\section{Conclusions}

The purpose of this paper is to study the existence of multiplicity solutions for fractional $p$-Laplacian equations with sublinear growth and oscillatory behavior. The key point is the choice of the framework to study the existence of weak solutions. In the suitably chosen framework, we are able to fulfil our strategy and generalize the corresponding results for fractional Laplacian equations. 


\section{Acknowledgements}

The author is grateful to anonymous reviewers for carefully reading this paper and for their comments and suggestions which have improved the paper.

\section{Funding}

The work is financially supported by the National Natural Science Foundation of China (Grant No. 11371110).

\section{Conflict of interest}

The author declares that there is no conflict of interest regarding the publication of this paper.

\section{Competing interests}

The author declares that he has no competing interests.

\section{Authors' contributions}

The author contributed wholly in writing this paper. He read and approved the final manuscript.

\section{Publisher's Note}

Springer Nature remains neutral with regard to jurisdictional claims in published maps and institutional affiliations.

Received: 6 July 2017 Accepted: 10 November 2017 Published online: 17 November 2017

\section{References}

1. Stein, EM: Singular Integrals and Differentiability Properties of Functions. Princeton Mathematical Series, vol. 30. Princeton University Press, Princeton (1970)

2. Applebaum, D: Lévy processes - from probability to finance quantum groups. Not. Am. Math. Soc. 51, 1336-1347 (2004)

3. Silvestre, LE: Regularity of the obstacle problem for a fractional power of the Laplace operator. Ph.D. Thesis, The University of Texas at Austin (2005)

4. Bucur, C, Valdinoci, E: Nonlocal Diffusion and Applications. Lecture Notes of the Unione Matematica Italiana, vol. 20. Springer, Bologna (2016)

5. Servadei, R, Valdinoci, E: Mountain Pass solutions for non-local elliptic operators. J. Math. Anal. Appl. 389, 887-898 (2012)

6. Servadei, R, Valdinoci, E: Variational methods for non-local operators of elliptic type. Discrete Contin. Dyn. Syst. 33(5), 2105-2137 (2013)

7. Xiang, M, Zhang, B, Ferrara, M: Existence of solutions for Kirchhoff type problem involving the non-local fractional p-Laplacian. J. Math. Anal. Appl. 424(2), 1021-1041 (2014)

8. Di Castro, A, Kuusi, T, Palatucci, G: Local behavior of fractional p-minimizers. Ann. Inst. H. Poincaré (C) Anal. Non Linéaire 33(5), 1279-1299 (2015)

9. Pucci, P, Xiang, M, Zhang, B: Multiple solutions for nonhomogeneous Schrödinger-Kirchhoff type equations involving the fractional $p$-Laplacian in $\mathbb{R}^{N}$. Calc. Var. Partial Differ. Equ. 54, 2785-2806 (2015)

10. Radulescu, VD, Xiang, M, Zhang, B: Existence of solutions for a bi-nonlocal fractional $p$-Kirchhoff type problem. Comput. Math. Appl. 71(1), 255-266 (2015)

11. Iannizzotto, A, Liu, S, Perera, K, Squassina, M: Existence results for fractional $p$-Laplacian problems via Morse theory. Adv. Calc. Var. 9(2), 101-125 (2016)

12. Dávila, J, Del Pino, M, Dipierro, S, Valdinoci, E: Concentration phenomena for nonlocal equations with Dirichlet datum. Anal. PDE 8(5), 1165-1235 (2015)

13. Dipierro, S, Medina, M, Peral, I, Valdinoci, E: Bifurcation results for a fractional elliptic equation with critical exponent in $\mathbb{R}^{N}$. Manuscr. Math. 153(1-2), 183-230 (2017)

14. Dipierro, S, Medina, M, Valdinoci, E: Fractional Elliptic Problems with Critical Growth in the Whole of $\mathbb{R}^{N}$. Lecture Notes. Scuola Normale Superiore di Pisa (New Series), vol. 15. Edizioni della Nor-male, Pisa (2017)

15. Fu, Y, Pucci, P: Multiplicity existence for sublinear fractional Laplacian problem. Appl. Anal. 96(9), 1497-1508 (2016)

16. Bartsch, T, Liu, ZL: Multiple sign changing solutions of a quasilinear elliptic eigenvalue problem involving the p-Laplacian. Commun. Contemp. Math. 6, 245-258 (2004)

17. Bartsch, T, Liu, ZL: Location and critical groups of critical points in Banach spaces with an application to nonlinear eigenvalue problems. Adv. Differ. Equ. 9, 645-676 (2004)

18. Di Nezza, E, Palatucci, G, Valdinoci, E: Hitchhiker's quide to the fractional Sobolev spaces. Bull. Sci. Math. 136, 521-573 (2012)

19. Bartsch, T, Liu, Z: On a superlinear elliptic p-Laplacian equation. J. Differ. Equ. 198(1), 149-175 (2004)

20. Iannizzotto, A, Mosconi, S, Squassina, M: Global Hölder regularity for the fractional p-Laplacian. Rev. Mat. Iberoam. 32, 1353-1392 (2016)

21. Del Pezzo, LM, Quaas, A: A Hopf's lemma and a strong minimum principle for the fractional p-Laplacian. J. Differ. Equ. 263(1), 765-778 (2017)

22. Kinderlehrer, D, Stampacchia, G: An Introduction to Variational Inequalities and Their Applications. Pure and Applied Mathematics, vol. 88. Academic Press, New York (1980)

23. Zeidler, E: Nonlinear Functional Analysis and Its Applications, II/B, Nonlinear Monotone Operators. Springer, New York (1990)

24. Willem, M: Minimax Theorems. Progress in Nonlinear Differential Equations and Their Applications, vol. 24. Birkhäuser, Boston (1996)

25. Rudin, W: Real and Complex Analysis. McGraw-Hill, New York (1966) 